\title{
The low-energy enhancements of the Pierre Auger Observatory
}

\author{
A.M. van den Berg* for the Pierre Auger Collaboration ${ }^{\dagger}$ \\ Kernfysisch Versneller Instituut, University of Groningen, NL-9747 AA Groningen, The \\ Netherlands \\ E-mail: bergekvi.nl
}

The Pierre Auger collaboration uses its observatory in Argentina to study ultra-high energy cosmic rays $(E>1 \mathrm{EeV})$. The baseline detectors are a surface detector array with 1660 waterCherenkov detectors distributed over an area of $3000 \mathrm{~km}^{2}$ overseen by 24 fluorescence telescopes mounted at the perimeter of this array. Recently, the collaboration has extended its observatory with three new detection systems, which will lower the energy threshold of the baseline detectors from about $1.0 \mathrm{EeV}$ to about $0.1 \mathrm{EeV}$. With this threshold we can study the region where we expect the transition from a Galactic to an extra-Galactic origin of cosmic rays and we can study particle-particle interactions in the $\mathrm{TeV}$ range. The extensions are AERA (Auger Engineering Radio Array), AMIGA (Auger Muon and Infill for the Ground Array), and HEAT (High Elevation Auger Telescopes), which will work side-by-side, bringing together for the very first time these complementary detection techniques. The information from these systems will be used to determine in detail the flux spectrum and to address the composition of cosmic rays with $\mathrm{E}>0.1$ $\mathrm{EeV}$. The current status of, and initial results from, these three detection systems are discussed.

The 2011 Europhysics Conference on High Energy Physics-HEP 2011,

July 21-27, 2011

Grenoble, Rhône-Alpes France

\footnotetext{
*Speaker.

${ }^{\dagger}$ Full author list available at http://www.auger.org/archive/authors_2011_07.html
} 


\section{Introduction}

After 100 years of research in cosmic-ray physics, some very fundamental questions have still not yet been answered. Where do these cosmic rays come from? How can they be accelerated to extreme energies at the EeV scale? What is the nature or composition of these particles? The detailed registration of the avalanche of secondary particles, known as an air shower, is an essential tool to infer properties of the primary particle, such as its energy, its arrival direction, and its composition. At this moment, the preferred method to assess the nature of the primary particle is based on the determination of the longitudinal shower profile, for instance by using optical detectors which collect the ultra-violet photons emitted by excited nitrogen molecules along the path of the shower. As this light signal is very weak, it can only be observed during dark periods, limiting the uptime for this detection technique to about $10 \%$. Obviously, this affects the detection statistics. Surface-detector arrays also have sensitivity to the composition of the primary cosmic ray. The baseline detectors of the Pierre Auger Observatory is an array of 1660 water-Cherenkov detectors distributed over an area of $3000 \mathrm{~km}^{2}$ overseen by 24 fluorescence telescopes mounted at four sites on the perimeter of this array; composition studies with these baseline detector systems are reported by Wahlberg [1]. Because of the relatively small cosmic-ray flux at very high energies $(E>50 \mathrm{EeV})$ and because of the poor detection efficiency at relatively low energies $(E<1 \mathrm{EeV})$, limited data are available to study the details of the mass composition in these two energy domains. Therefore, the observatory is being enhanced with additional detector systems. As a first step, the region between 0.1 and $1.0 \mathrm{EeV}$ is being explored with an extension of the existing techniques (fluorescence detection and water-Cherenkov detection) at a section of the observatory while, in addition, other and new detection techniques are being explored using underground muon counters and radio receivers, respectively. This energy range is of particular importance, because it is thought to be the region where the transition may take place from a Galactic to an extra-Galactic origin of cosmic rays. Of course, the transition point may strongly depend on the composition of the cosmic rays in the energy range.

\section{AERA}

In an air shower, copious numbers of electrons and positrons are created forming a pancakeshaped cloud with a typical thickness of less than $1 \mathrm{~m}$. The geomagnetic field induces a drift velocity which is perpendicular to the direction of the initial cosmic ray and which is opposite for electrons and positrons. The strength of this current is roughly proportional to the number of charge carriers. Because the number of charge carriers changes rapidly as the shower progresses through the atmosphere, there is a corresponding change in the induced current. This leads to the coherent emission of electromagnetic waves at wavelengths longer than the size of the dimension of the charge cloud, i.e. for radio frequencies smaller than about $300 \mathrm{MHz}$, which is the VHF band. Thus, radio signals can be used to probe the increase and the decrease of the number of electrons and positrons in the shower. The dominant contribution for radio emission is caused by electrons and positrons while their energy is in the $\mathrm{MeV}$ range. This is in contrast to the emission of fluorescence light, which is mainly caused by interactions of low energy electrons with nitrogen molecules. Therefore, radio signals carry information which is complementary to that from the 
observation by fluorescence emission as well as to that from the detection of secondary particles hitting the surface of the Earth.

The scientific goals of the AERA project are as follows: 1) calibration of the radio emission from the air showers, including sub-dominant emission mechanisms; 2) demonstration at a significant scale of the physics capabilities of the radio technique, e.g. energy, angular, and primary mass resolution; and 3) measurement of the cosmic-ray composition from 0.3 to $5 \mathrm{EeV}$, with the goal of elucidating the transition from Galactic to extra-Galactic cosmic rays. Results from prototype versions of AERA and a description of its present configuration are given by Refs. [2, 3], respectively. Here we mention only that in its present configuration each detection station consist of a dual-polarization (north-west and east-south) log-periodic dipole antenna, associated analog and digital readout electronics, an autonomous power system, and a high-speed fiber-optical communications link to a central data acquisition system. The antennas are sensitive between 27 and $84 \mathrm{MHz}$, chosen as the relatively radio-quiet region between the shortwave and FM bands. AERA deployment began in 2010 in order to address these technical and scientific issues. Stable physics data-taking started in March 2011, and the first hybrid detection of cosmic-ray events by radio, fluorescence, and surface particle detectors have been recorded in April 2011.

\section{AMIGA and HEAT}

The low-energy extension of the fluorescence detector of the observatory are the High Elevation Auger Telescopes (HEAT [4]), which operate on the same principles as one of the standard fluorescence telescopes of the observatory. HEAT has been designed to register relatively low energy showers for which the shower maximum is on the average higher in the atmosphere as compared to showers induced by cosmic rays with $E>1 \mathrm{EeV}$. Therefore, these telescopes cover an angular range between $29^{\circ}$ and $58^{\circ}$ above the horizon. HEAT has been in operation since September 2009 and is producing high quality data, leading to a dramatic improvement in the determination of the cosmic-ray composition at relatively low energies. The low-energy extension of the surface detector of the observatory is the Auger Muon and Infill for the Ground Array (AMIGA [5]), which consists of additional water-Cherenkov surface detectors placed on a denser grid as compared to the regular surface detector grid. Each of the 85 detection stations in this low-energy extension will be equipped with additional scintillation counters buried at a depth of $2.3 \mathrm{~m}$ acting as muon counters. As of April 2011, 53 of the 85 extra water-Cherenkov tanks had been deployed and are currently producing reliable data.

\section{References}

[1] H. Wahlberg [Pierre Auger Collaboration], this conference

[2] B. Revenu [Pierre Auger Collaboration], 32nd ICRC, Beijing, China, August, 2011; arXiv:1107.4807

[3] J. Kelley [Pierre Auger Collaboration], 32nd ICRC, Beijing, China, August, 2011; arXiv:1107.4807

[4] T. Hermann-Josef Mathes [Pierre Auger Collaboration], 32nd ICRC, Beijing, China, August, 2011; arXiv:1107.4807

[5] F. Sánchez [Pierre Auger Collaboration], 32nd ICRC, Beijing, China, August, 2011; arXiv:1107.4807 